\title{
Textiloma in leg - A Diagnostic Quandary
}

\author{
Agarwal $\mathrm{A}^{* 1}$, Sharma $\mathrm{M}^{2}$, and Nanda $\mathrm{V}^{3}$ \\ ${ }^{1}$ Associate consultant - Department of Cosmetic and Plastic surgery, Artemis hospital, Gurgaon, India \\ ${ }^{2}$ Senior Consultant - Department of Cosmetic and Plastic surgery, Artemis hospital, Gurgaon, India \\ ${ }^{3}$ Senior Consultant and Head - Department of Cosmetic and plastic surgery, Artemis hospital, Gurgaon, India
}

${ }^{*}$ Corresponding author: Agarwal A, Associate consultant - Department of Cosmetic and Plastic surgery, Artemis hospital, Gurgaon, India, Tel: 91 8800274318, E-mail: avindian80@gmail.com

Citation: Agarwal A, Sharma M, Vipul N (2018) Textiloma in leg - A Diagnostic Quandary. J Surg Oper Care 3(1): 103. doi: 10.15744/2455-7617.3.103

Received Date: February 23, 2018 Accepted Date: May 3, 2018 Published Date: May 7, 2018

\begin{abstract}
Objective: To report a case of leg textiloma and highlight the different clinical presentations and preventive measures.

Case report and intervention: A 28 year old male patient presented with multiple discharging sinuses in the right leg. He had a history of surgery 1 year ago. On exploration a ball of cotton fibre encapsulated by scar tissue was found engulfed in the medial belly of the gastrocnemius muscle.

Conclusion: Textiloma is a preventable complication. It may present a diagnostic dilemma due to its non specific imaging features and different clinical presentations. A strict gauze count protocol should be followed to avoid this.

Keywords: Gossypiboma; Textiloma
\end{abstract}

\section{Introduction}

A variety of agents are used during any surgical procedure to control intraoperative bleeding. Most of them are meant to be removed at the end of the procedure. Sometimes these are accidentally forgotten at the operative site which may present with different clinical symptoms subsequently.

Textiloma, gossypiboma or muslinoma are various terms used for this entity. Reported estimate of all the retained foreign bodies (RFB) is 1 in 1000 to 1 in 10,000 [1] and in 80 percent of them a surgical sponge is the culprit [2]. The reported incidence of gossypiboma varies from one per 300- 1000 for all the surgical procedures [3].This is probably an underestimate due to underreporting because of medico legal consequences. Most of such cases are reported in the abdominal cavity but the chest, the extremity and the cranial cavity have also been reported [4]. We report one such case in the leg.

\section{The case}

A 28 year old male presented to emergency plastic surgery department with complaints of multiple discharging sinuses on the posterior aspect of the right leg for the last 8 months. He had a history of injury to the right leg in a blast about 1 year back for which he was operated (details not available). He was again operated 4 months later for an abscess at the operative site after which he developed multiple discharging sinuses in the lower third the right leg (Figure 1).

On examination there were two sinuses discharging purulent material. There was associated dense scarring in the surrounding tissues. An X ray and MRI was done to look for underlying osteomylitis, any abscess or soft tissue growth.MRI was suggestive of a well defined,walled off collection in the posterior aspect of the right leg in the intramuscular plane extending upto the subcutaneous plane (Figure 2).

This information was shared with the patient and his family was counseled about the plan.

On exploration, a ball of cotton fibres encapsulated by scar tissue was seen engulfed in the medial belly of the Gasrtocnemius muscle with ramifications of pus pockets all around, two of it forming cutaneous sinuses. Gossypiboma was excised along with the scarred tissue and the involved fibres of muscle belly and tendo achiles. (Figure 3) The wound cavity was closed in layers over a drain which was removed on third post operative day. 


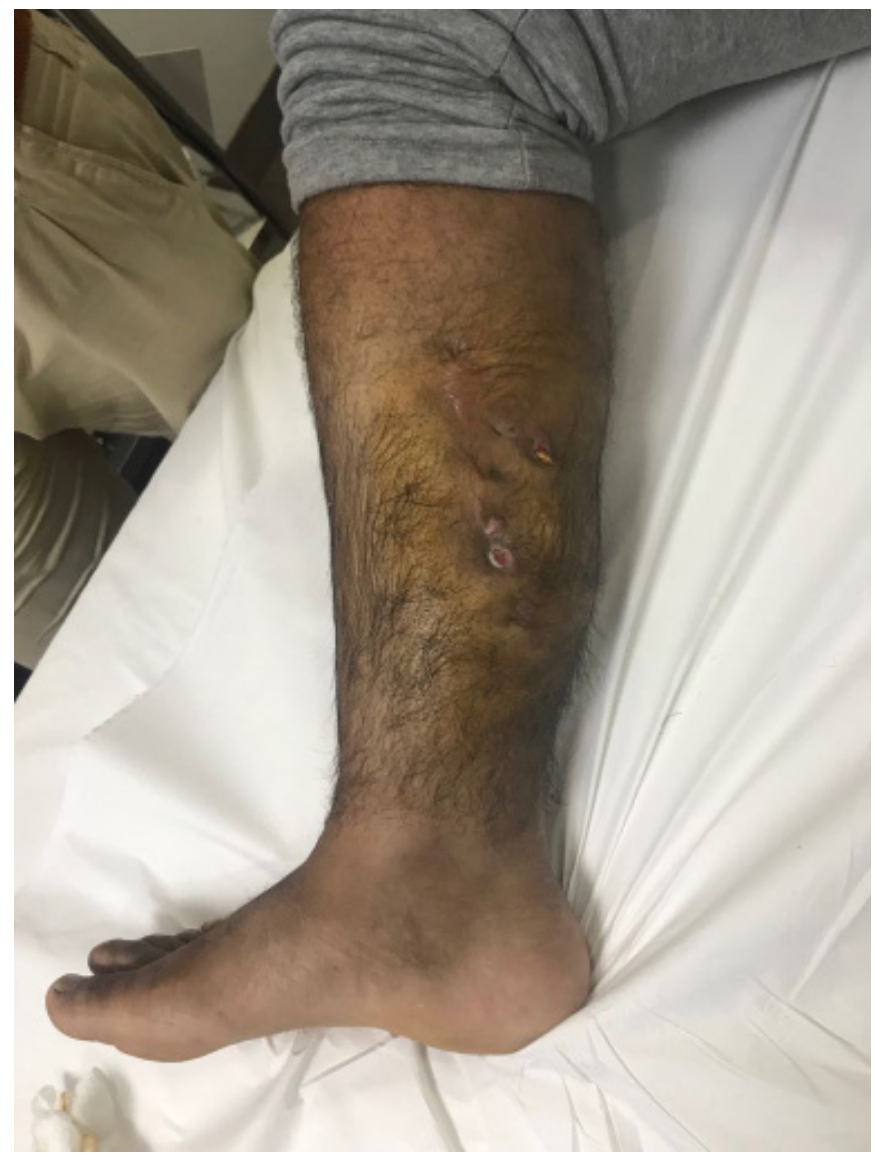

Figure 1: Pre-Operative Presentation

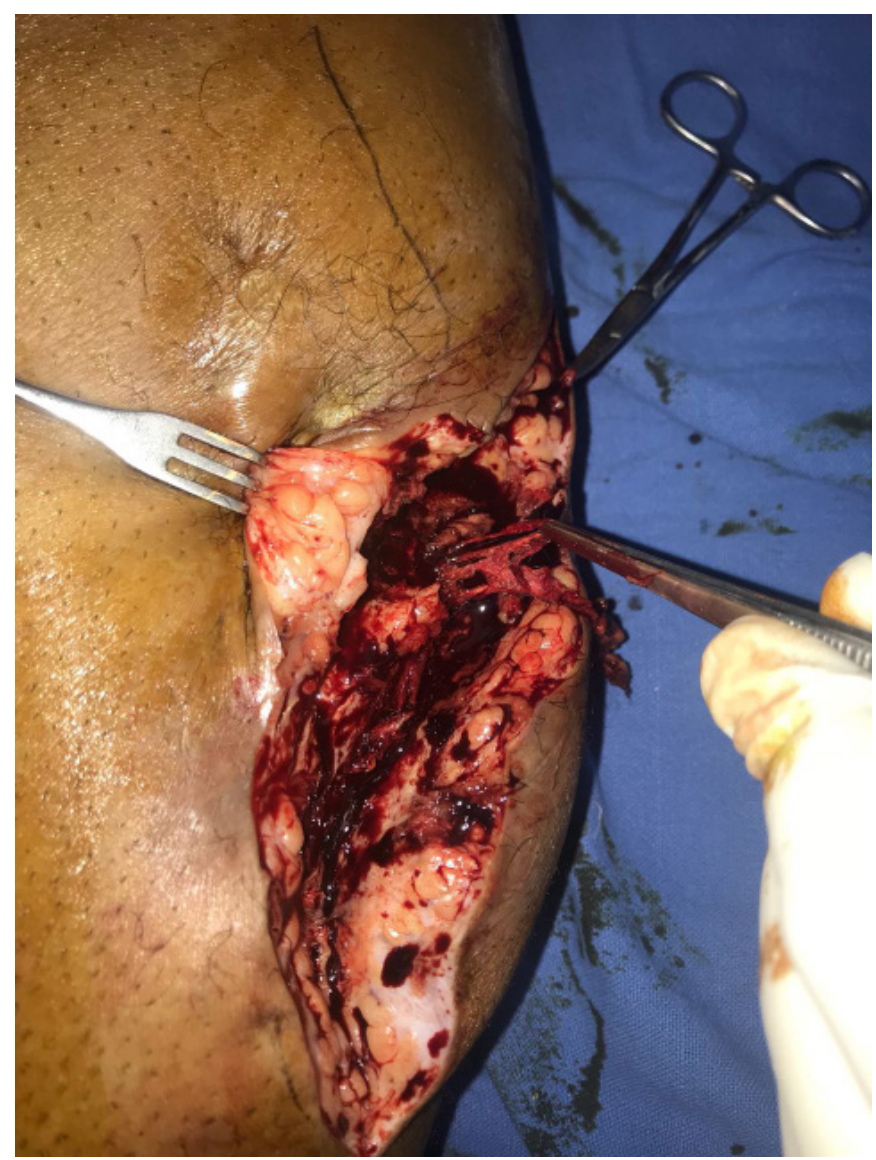

Figure 2: Intra operative finding textiloma being removed

Histopathology showed formation of acute over chronic inflammatory granulation tissue and presence of many foreign body giant cells around thread like foreign material. No evidence of granuloma or malignancy was seen. 


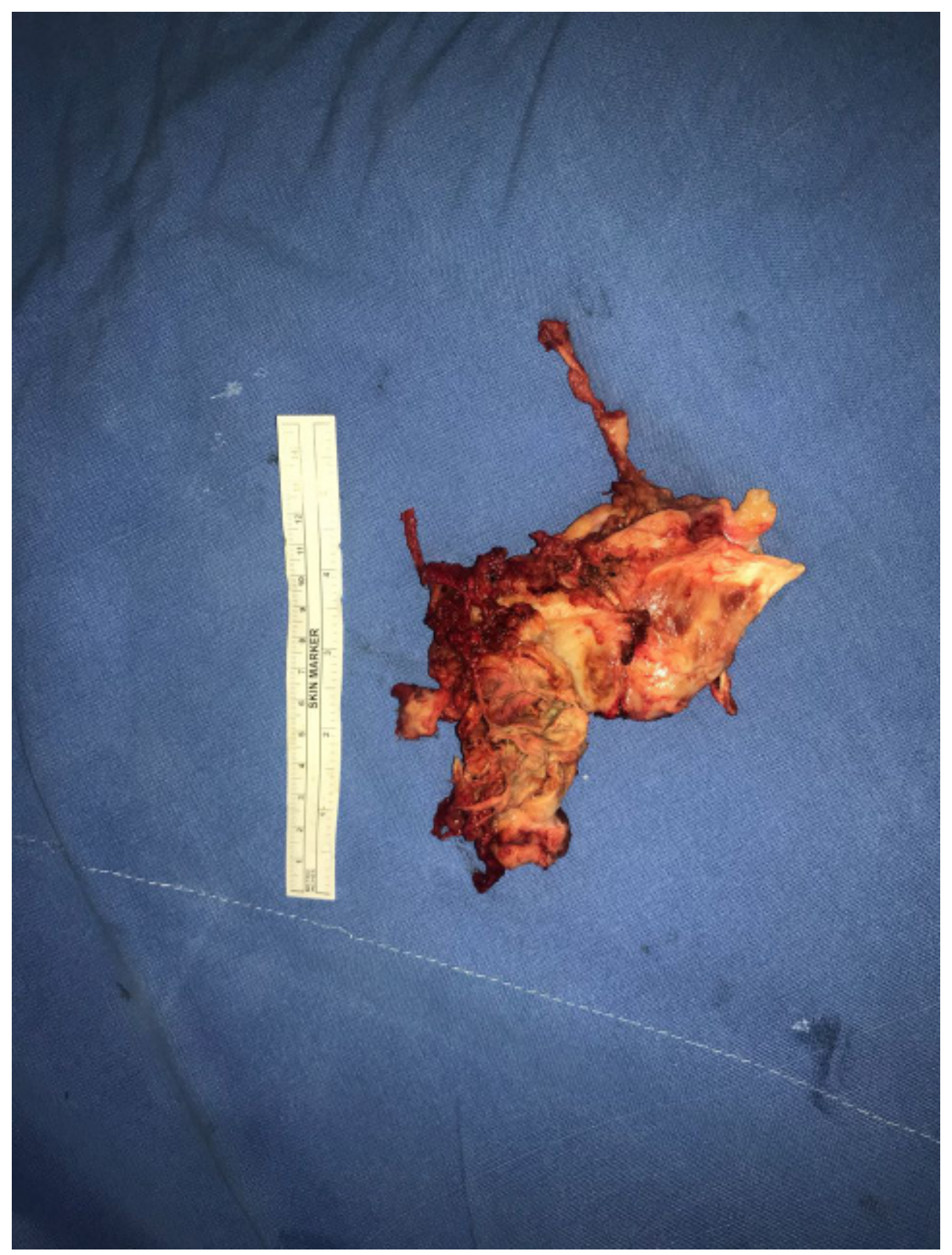

Figure 3: Excised foreign body from the wound

At 6 weeks follow up the wound is well healed and the soft tissues of the leg appears supple.

\section{Discussion}

Textiloma or gossypiboma is a term used to define a retained foreign body after surgery, most commonly a gauze piece. The first such case was reported in 1884 by Wilson [5]. The incidence of this entity varies from one in 1000- 1500 for intra abdominal operations and one per 300- 1000 for all the surgeries [3]. The true incidence is difficult to measure due to under reporting because of its medicolegal implication. Diagnosis of gossypiboma or retained foreign body, after any surgical procedure, has often led to litigations and huge amount of compensation and legal cost expenses [6].

Various absorbable and non absorbable materials are used to control intra operative bleeding. These may be left inside intentionally or unintentionally. This leads to an acute inflammatory reaction around that material which subsides with resoprtion of this material. At times, exuberant inflammatory reaction leads to formation of pseudo capsule around this foreign body. The type of reaction determines the presentation of the patient. It may be exudative leading to discharging sinuses, fistulas and abscesses or a dense fibrosis presenting as a pseudomotor [7].

Diagnosis of gosspiboma is difficult to due to its nonspecific clinical features .A history of previous surgery may provide a clue. It should be kept in mind that the time interval between surgery and presentation may be as long as up to 15 years [8]. Features of various imaging modalities are also not very specific. Features such as Radio opaque marker or a whirl like image on $\mathrm{X}$ ray (35\%), hyper echoic mass with hypo echoic rim on ultrasound (34\%) and presence of a round mass with a dense central part and enhancing rim on CT scan (61\%) may be helpful in assessment [3]. 
Gossypiboma is a totally preventable complication which requires a religious vigil on the mops and gauze pieces used during the surgery [1]. It's a good idea to keep the other end of the mop outside whenever possible. Counting the mops before and after the procedure should be done to prevent this complication. The surgeon should perform methodical wound exploration before closure of any anatomical cavity or the surgical site. Counts should be done for any procedure in which sponges, sharps, miscellaneous items or instruments could be retained in the patient. These counts must be performed at least at the beginning and end of every eligible case. Validated, automated sponge counting systems, such as bar coded or radio labeled sponges, should be considered for use when feasible [9]. Counting should be checked again before closing the abdominal cavity. In case the cavity is packed during the surgery, exact number and type of material should be documented. One should always avoid the use of loose cotton for packing.

In post operative period persistence of pain, erythema or discharge may point to the diagnosis.

\section{Conclusion}

Although rare, a retained foreign body following any surgical intervention should be considered a probability when presented with a similar situation. We should strictly follow the procedure of gauze count during surgery. Validated, automated sponge counting systems, such as bar coded or radio labeled sponges, should be considered for use when feasible.

\section{References}

1. Patel AC, Kulkarni GS, Kulkarni SG (2007) Textiloma in the leg. Indian J Orthop 41: 237-8.

2. Patial T, Thakur V, Vijhay Ganesun N, Sharma M (2017) Gossypibomas in India - A systematic literature review. J Postgrad Med 63: 36.

3. Naeem M, Ahmad M, Samad A, ul Haq I (2017) GOSSYPIBOMA: A medical negligence. Pak J Surg 33: 83-6.

4. Pole G, Thomas B (2017) A Pictorial Review of the Many Faces of Gossypiboma - Observations in 6 Cases. Pol J Radiol 82: 418-21.

5. Mouhsine E, Garofalo R, Cikes A, Leyvraz PF (2006) Leg Textiloma. Med Princ Pract 15: 312-5.

6. Gawande AA, Studdert DM, Orav EJ, Brennan TA, Zinner MJ (2003) Risk factors for retained instruments and sponges after surgery. N Engl J Med 348: 229-5.

7. Sharma G, Bigelow J (2014) Retained foreign bodies: A serious threat in the Indian operation room. Ann Med Health Sci Res 4: 30.

8. Rehman A, Baloch NU-A, Awais M (2014) Gossypiboma diagnosed fifteen years after a cesarean section: A case report. Qatar Med J 2014 : 12.

9. World Health Organization, Patient Safety (2009) WHO guidelines for safe surgery 2009: safe surgery saves lives.

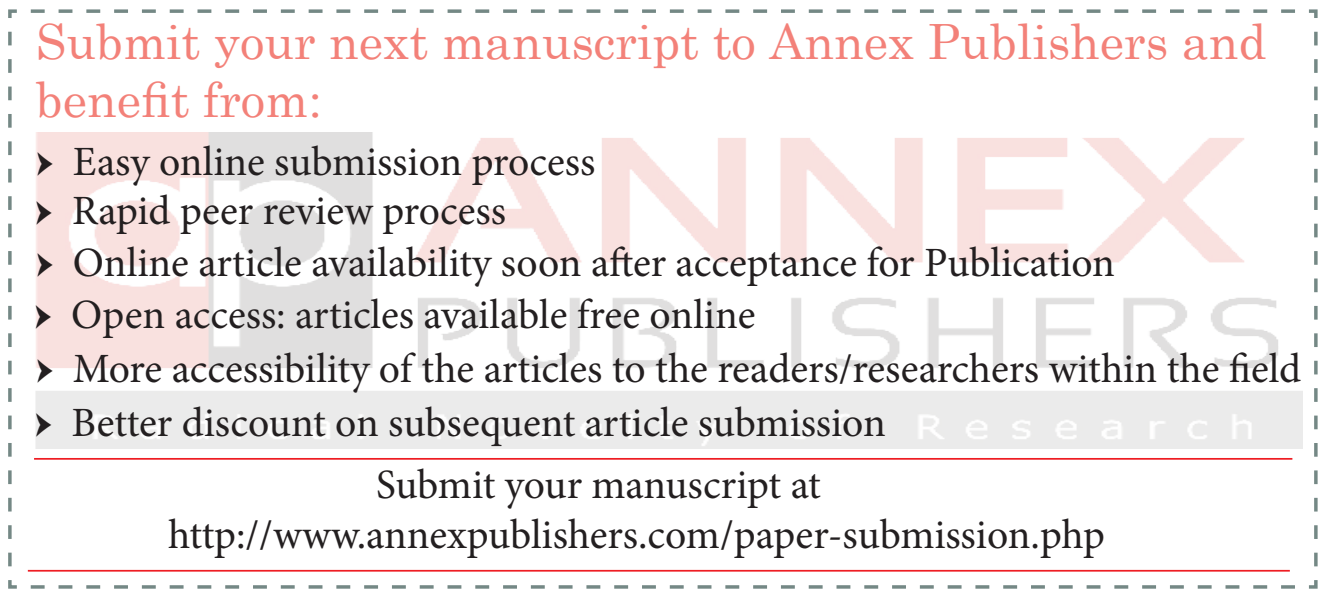

